\author{
Carmen Andrea Pfortmueller \\ Gregor Lindner \\ Alexander Benedikt Leichtle \\ Georg Martin Fiedler \\ Aristomenis Konstantinos \\ Exadaktylos
}

\section{Diagnostic significance of high sensitivity troponin in diagnosis of blunt cardiac injury}

Accepted: 20 December 2013

Published online: 25 January 2014

(C) Springer-Verlag Berlin Heidelberg and ESICM 2014

Carmen Andrea Pfortmueller and Gregor Lindner have contributed equally to this work.

Electronic supplementary material The online version of this article (doi:10.1007/s00134-013-3204-5) contains supplementary material, which is available to authorized users.

Dear Editor,

Blunt cardiac injury (BCI) is commonly associated with major thoracic trauma and influences morbidity and mortality to a great extent $[1,2]$.

Despite much discussion in recent years, no diagnostic "gold standard" exists. Therefore the primary aim of this study was to evaluate the diagnostic significance of high sensitivity troponin measurements in patients with BCI. In our cross-sectional analysis, all patients admitted with trauma were included for whom high sensitivity serial measurements had been performed of troponin $\mathrm{T}$ between 1 August 2010 and 31

October 2012 at the Department of

Emergency Medicine (ED),

Inselspital.

Blunt cardiac trauma was suspected in 31 of 86 patients admitted with trauma. For an overview of patient characteristics see Table 1 . The median injury severity score (ISS) and injury count were significantly higher in patients with BCI (9.13, SD 9.53, $p<0.001$ and 4.71, SD 2.5, $p<0.0001$, respectively). The difference between the first and second high sensitivity troponin $\mathrm{T}$ measurements was significantly higher in patients with clinically suspected BCI than in others $(0.003 \mu \mathrm{g} / \mathrm{l}$, interquartile range (IQR) $0.00-0.12$ and $0.002 \mu \mathrm{g} / \mathrm{l}$, IQR $0.00-0.005$, $p<0.001$, respectively). Neither ECG changes of any type nor arrhythmia or cardiac shock correlated with the high sensitivity troponin $\mathrm{T}$ value on admission $(p<0.14, p<0.25, p<0.35$, respectively) or at $3 \mathrm{~h}(p<0.24$, $p<0.65, p<0.33$, respectively) or with the change in the high sensitivity troponin $\mathrm{T}$ value $(p<0.76, p<0.54$, $p<0.41$, respectively). The change from the first to the second high sensitivity troponin $\mathrm{T}$ measurement was positively correlated with the ISS $(p<0.04)$. An increase of $20 \%$ or more in high sensitivity troponin $\mathrm{T}$ has a sensitivity of $54.8 \%$, a specificity of $69.1 \%$ and a positive likelihood ratio of 1.77 for clinically suspected BCI. The combination of an increase in high sensitivity troponin $\mathrm{T}$ of more than $20 \%$ and $\mathrm{ECG}$ changes has a sensitivity of $64.5 \%$ and a specificity of $53.3 \%$ and a positive likelihood ratio of 1.36 for clinically suspected BCI.

Patients without BCI less often have positive high sensitivity troponin $\mathrm{T}$ values at the second measurement than patients with clinically suspected BCI. This pattern is explained by the prolonged release of the protein from injured myocardial cells [2]. It has been reported that, after severe myocardial damage, protein release may be prolonged by myocardial hypoxia caused by microvascular injury $[2,3]$. Nevertheless and contrary to other studies on conventional troponin $\mathrm{T}[1,2]$, patients in our study with and without clinically suspected BCI did not significantly differ in baseline high sensitivity troponin $\mathrm{T}$ values. This may result from the lower specificity of high sensitivity troponin $\mathrm{T}$ for myocardial necrosis compared to conventional troponin T [4]. Even though high sensitivity troponin $\mathrm{T}$ measurements have 10- to 15 -fold higher sensitivity for detection of myocardial damage than conventional troponin measurements, they more often detect elevated values caused by non-ischemic causes such as sepsis, pulmonary embolus or renal failure $[4,5]$.

Our findings suggest that high sensitivity troponin T measurement in trauma patients is a reliable biologic marker for estimating myocardial injury. But because of its low specificity, not the actual value but more the dynamics should be considered 
Table 1 Patients characteristics

\begin{tabular}{|c|c|c|c|c|}
\hline & Total $(N, \%)$ & Suspected BCI $(N, \%)$ & No suspected $\mathrm{BCI}(N, \%)$ & $p$ value \\
\hline$N$ & $86(100)$ & $31(36.0)$ & $55(64.0)$ & \\
\hline Male/female & $61(70.9) / 25(29.1)$ & $25(41.0) / 6(24.0)$ & $36(59.0) / 19(76.0)$ & 0.21 \\
\hline Age (median, range) & $71(19-94)$ & $63(19-93)$ & $79(20-94)$ & 0.089 \\
\hline \multicolumn{5}{|l|}{ Type of accident } \\
\hline Traffic accident & $29(33.7)$ & $20(68.9)$ & $9(31.1)$ & 0.0001 \\
\hline Fall & $57(66.3)$ & $11(19.3)$ & $46(80.7)$ & 0.0001 \\
\hline \multicolumn{5}{|l|}{ Mean AIS (mean, SD) } \\
\hline Thorax & $1.77(0.71)$ & $1.77(0.71)$ & & * \\
\hline Abdomen & $2.25(1.25)$ & $3.0(1.41)$ & $1.50(0.7)$ & 0.0001 \\
\hline Head & $1.43(0.78)$ & $1.07(0.25)$ & $1.52(0.94)$ & 0.0001 \\
\hline Face & $1.32(0.47)$ & $1.13(0.35)$ & $1.39(0.49)$ & 0.001 \\
\hline Spine & $2.71(1.38)$ & $3.60(0.89)$ & $2.22(1.39)$ & 0.045 \\
\hline Upper extremities & $1.38(0.66)$ & $1.56(0.88)$ & $1.25(0.45)$ & 0.015 \\
\hline Lower extremities & $1.93(0.78)$ & $2.00(0.81)$ & $1.9(0.78)$ & 0.89 \\
\hline External & $1.14(0.53)$ & $1.50(1.00)$ & $1.00(0.00)$ & 0.0001 \\
\hline ISS (median, range) & $4(1-41)$ & $9.13(9.53)$ & $5.44(4.77)$ & 0.001 \\
\hline ECG changes at admission & $21(24.4)$ & $5(23.8)$ & $16(76.2)$ & 0.2 \\
\hline \multicolumn{5}{|l|}{ Hospitalisation } \\
\hline Outpatient/inpatients & $46(46.5) / 40(53.5)$ & $15(37.5) / 16(34.8)$ & $25(62.5) / 30(65.2)$ & 0.82 \\
\hline \multirow[t]{2}{*}{ In-hospital mortality } & $3(3.5)$ & $1(33.3)$ & $2(66.7)$ & 0.92 \\
\hline & Total (median, IQR) & Suspected BCI (median, IQR) & No BCI (median, IQR) & $p$ value \\
\hline Troponin $1(\mu \mathrm{g} / \mathrm{l})$ & $0.016(0.005-0.027)$ & $0.011(0.004-0.24)$ & $0.016(0.006-0.027)$ & 0.27 \\
\hline Troponin $2(\mu \mathrm{g} / \mathrm{l})$ & $0.021(0.008-0.037)$ & $0.019(0.006-0.055)$ & $0.022(0.012-0.037)$ & 0.008 \\
\hline Delta troponin $(\mu \mathrm{g} / \mathrm{l})$ & $0.0020(0.00-0.006)$ & $0.003(0.00-0.12)$ & $0.002(0.00-0.005)$ & 0.001 \\
\hline Creatinine kinase (U/l) & $187(88.25-411.00)$ & $192.5(99.5-423.75)$ & $165(74.0-341.75)$ & 0.11 \\
\hline MDRD & $73.00(60.0-91.50)$ & $86.5(63.5-96.25)$ & $71.0(58.0-84.0)$ & 0.81 \\
\hline
\end{tabular}

MDRD modification of diet in renal disease

* Only two per category

when assessing patients with suspected $\mathrm{BCI}$.

Conflicts of interest None.

Ethical standard The study was approved by the regional ethics committee.

\section{References}

1. Helm M, Hauke J, Weiss A, Lampl L (1999) Cardiac troponin $\mathrm{T}$ as a biochemical marker of myocardial injury early after trauma. Diagnostic value of a qualitative bedside test. Chirurg 70:1347-1352

2. Rajan GP, Zellweger R (2004) Cardiac troponin I as a predictor of arrhythmia and ventricular dysfunction in trauma patients with myocardial contusion. J Trauma 57:801-808 (discussion 808)
3. Swaanenburg JC, Klaase JM, DeJongste MJ, Zimmerman KW, ten Duis HJ (1998) Troponin I, troponin T, CKMBactivity and CKMB-mass as markers for the detection of myocardial contusion in patients who experienced blunt trauma. Clin Chim Acta 272:171-181

4. Wu AH, Jaffe AS (2008) The clinical need for high-sensitivity cardiac troponin assays for acute coronary syndromes and the role for serial testing. Am Heart J 155:208-214

5. Bessiere F, Khenifer S, Dubourg J, Durieu I, Lega JC (2013) Prognostic value of troponins in sepsis: a metaanalysis. Intensive Care Med 39:1181-1189

C. A. Pfortmueller $(\bullet) \cdot$ G. Lindner Department of General Internal Medicine, University Hospital and University of Bern, 3010 Bern, Switzerland e-mail: cpfortmueller@gmail.com
G. Lindner

e-mail: lindner.gregor@gmail.com

A. B. Leichtle - G. M. Fiedler

Center of Laboratory Medicine, University Institute of Clinical Chemistry, InselspitalBern University Hospital, Inselspital, Bern, Switzerland

e-mail: benedikt.leichtle@insel.ch

G. M. Fiedler

e-mail: georg.fiedler@insel.ch

C. A. Pfortmueller - G. Lindner .

A. K. Exadaktylos

Department of Emergency Medicine,

University Hospital and University of Bern, 3010 Bern, Switzerland

e-mail: exadaktylos@exadaktylos.ch 